\title{
Procedimientos de creación léxica en las designaciones iberorrománicas del párpado $(\mathrm{I})^{1}$
}

\author{
Carolina JULiÀ LUNA \\ Departamento de Filología Española \\ Universitat Autònoma de Barcelona \\ carolina.julia@uab.cat
}

\section{RESUMEN}

El objetivo de la presente investigación ${ }^{2}$ es el estudio de las denominaciones del párpado en las lenguas iberorrománicas a partir del examen de los datos que contienen los atlas lingüísticos del español, el catalán, el gallego y el portugués. Los resultados obtenidos reflejan el valor de los materiales sobre el léxico iberorrománico que contienen los atlas lingüísticos para dar cuenta de que la metáfora y la metonimia son los dos mecanismos semánticos más frecuentes en los procesos de creación del léxico popular.

Palabras clave: metáfora, metonimia, geografía lingüística, lenguas iberorrománicas

[Recibido, septiembre 2010; aprobado, diciembre 2010]

\author{
Some procedures of lexical creation \\ in the Ibero-Romance names of the 'eyelid' (I)
}

\begin{abstract}
This research's goal is to study the names of the 'eyelid' in the Iberio-Romance languages, after having examined the data contained in the linguistic atlases of Spanish, Catalan, Galician and Portuguese. The results allow us to say how valuable the linguistic atlases are in order to consider metaphor and metonymy as the two most frequent procedures of popular lexical creation.
\end{abstract}

Keywords: metaphor, metonymy, linguistic geography, Ibero-Romance languages

\section{Introducción}

Los atlas lingüísticos constituyen grandes corpus de datos en los que, además de recogerse referencias geográficas sobre el uso y la vitalidad de las palabras, se reúnen numerosas informaciones fonéticas, morfosintácticas y léxico-semánticas

\footnotetext{
${ }^{1}$ Por cuestiones editoriales, el artículo se ha dividido en dos partes; en la primera, se presenta la investigación y el análisis geolingüístico de los datos, y en la segunda, que aparecerá en el próximo número, se incluye el estudio etimológico y semántico de las denominaciones del párpado.

${ }^{2}$ El desarrollo de esta investigación ha sido posible gracias a la ayuda de la DGICYT (n. ${ }^{\circ}$ de ref. FFI2008-06324-C02-01) y del Comissionat per Universitats $i$ Recerca de la Generalitat de Catalunya (n. ${ }^{\circ}$ de ref. SGR2009-1067).
} 
sobre la lengua oral. Las investigaciones sobre los mapas geolingüísticos dan cuenta de que el examen de estas informaciones permite, entre otros muchos aspectos, completar y contrastar informaciones lingüísticas de diverso tipo. Véanse, por ejemplo, las aportaciones que los datos de los mapas pueden ofrecer a la lexicología (Alvar 1982; García Mouton 1990, 2010; Navarro Carrasco 2001; Prat 2006; Gordón Peral y Ruhstaller 2008; Castañer 2009).

Desde la perspectiva de análisis léxico-semántica, la organización onomasiológica de los mapas resulta idónea para estudiar las relaciones que se establecen entre los conceptos y sus designaciones y, al mismo tiempo, por tanto, también para entender los modos de conceptualización de realidades concretas y cotidianas. Por ello, con el fin de contribuir a la determinación y descripción de los procedimientos semánticos de creación léxica más comunes de los nombres de partes del cuerpo (Tagliavini 1949; Brown y Witkowski 1981), se analiza el conjunto de denominaciones que se recogen en los atlas lingüísticos iberorrománicos sobre el 'párpado'. Se ha elegido este concepto porque los mapas en los que se halla recogen un elevado grado de variación designativa (más de 60 formas léxicas distintas) y porque posee una representación importante en la geolingüística iberorromance, pues se halla en el cuestionario de la mayoría de $\operatorname{atlas}^{3}$ de las variedades habladas en las Península Ibérica $(A L D C$ - 15; ALEA - V, 1200; ALEANR - VII, 948; ALeCMan - 273; ALEICan - II, 480; $A L C y L-1132^{4} ; A L G a-\mathrm{V}, 15 ; A L P I-585 \mathrm{y}$ $A L E P G-0440^{5}$ ). Los datos de los mapas se han analizado desde el punto de vista léxico-semántico a partir de la aplicación de las teorías de la lingüística cognitiva.

Las partes del cuerpo constituyen, como han advertido numerosos lingüistas (Martín-Municio 1992; Martins-Baltar y Calbris 1997, 2009, entre otros muchos), una importante fuente de creación y atracción universal de metáforas y metonimias. Por ello, la aplicación de los postulados de la semántica cognitiva (Johnson 1992[1987]; Peña Cervel 2001; Goschler 2005) al estudio de la creación de significados relativos al cuerpo resulta muy interesante ya que esta teoría lingüística se basa, primordialmente, en principios experiencialistas y en la renovación de los conceptos de metáfora y metonimia. El análisis de los mapas lingüísticos referidos al cuerpo humano desde esta perspectiva teórica, como se ha comprobado en investigaciones anteriores (Julià 2007, 2009a, 2010), permite determinar las realidades mediante las que más comúnmente suele comprenderse y conceptualizarse el

\footnotetext{
${ }^{3}$ El único atlas en el que no se halla el concepto es el ALECant.

${ }^{4}$ En algunos atlas, como es el caso del $A L C y L$, dadas las pocas variantes designativas recogidas, no se presentan cartografiados los datos en un mapa sino que aparecen en un listado anexo al volumen en el que se hallan los conceptos referidos al léxico del cuerpo humano.

${ }^{5}$ Quiero hacer constar mi agradecimiento al Dr. João Saramago (Universidade de Lisboa), quien en julio de 2007 me permitió consultar algunos datos del inédito $A L E P G$ que se analizan en este artículo y que me sirvieron para llevar a cabo parte del trabajo de investigación titulado Léxico y variación: los nombres de las partes del ojo (Julià 2007). Para más información sobre este atlas y el estado de su publicación, véase Saramago (2006).
} 
cuerpo humano, es decir, posibilita el examen de este ámbito nocional como concepto o dominio meta. Las investigaciones desde este punto de vista se ven favorecidas, además, por la organización onomasiológica de los datos geolingüísticos. Sin embargo, como ha demostrado Alinei en numerosas ocasiones (Alinei 1984, 1997, 2005), esto no significa que los atlas lingüísticos no permitan realizar estudios semasiológicos de un campo conceptual, sino que el modo en el que se estructuran las informaciones beneficia considerablemente las investigaciones en las que se analizan las formas léxicas en relación con el referente al que designan.

Mediante el estudio de las distintas formas de referirse al párpado que compilan los atlas iberorrománicos ${ }^{6}$ se pretende contribuir, en la medida de lo posible, a mostrar el grado de variación léxica que se da en las designaciones de algunas partes del cuerpo; dar cuenta de la información que subyace a los datos léxicos de los atlas para el análisis de la creación léxico-semántica de las lenguas; ejemplificar el funcionamiento de la lengua, es decir, evidenciar los mecanismos más relevantes en el proceso de creación de significado (la metáfora y la metonimia); y, finalmente, aportar datos sobre la idea de que la creación de nombres de partes del cuerpo sigue un patrón de formación regular en las lenguas iberorrománicas, como se ha demostrado que sucede para otros conceptos ('pupila', 'genitales masculinos', 'músculos', 'dedos de la mano') en otras lenguas del mundo (Tagliavini 1949; Brown y Witkowski 1981; Veny 2000; Caprini y Ronzitti 2007; Julià 2009a, 2009b, 2010).

\subsection{Metáfora y metonimia en el estudio del léxico}

Desde el surgimiento de la lingüística cognitiva, se ha considerado que la metáfora y la metonimia constituyen mecanismos esenciales en la explicación del origen y las causas del cambio semántico (Johnson 1992[1987]; Santos y Espinosa 1996; Dworkin 2006a) y en consecuencia, se conciben como medios vitales en la justificación de la existencia de la polisemia léxica (Sweetser 1990). El cognitivismo ha supuesto una revolución en la semántica y, más concretamente, en la semántica diacrónica, pues aporta herramientas a los lingüistas que permiten enriquecer, mejorar y confirmar las explicaciones sobre la naturaleza del cambio de significado de las palabras.

La metáfora y la metonimia, desde el punto de vista de la teoría cognitiva, son dos de los procedimientos a los que recurre con más frecuencia la cognición para generar conocimiento y comprender el mundo. En el caso de la metáfora, por ejemplo, la comparación de aquellas realidades que son ajenas, complejas o abstractas con otras que son más cercanas, conocidas o palpables es lo que permite al ser humano comprender y aprehender el entorno. Sirva de ejemplo la metáfora conceptual de tipo estructural EL TIEMPO ES DINERO (Lakoff y Johnson

\footnotetext{
${ }^{6}$ Los atlas examinados son los siguientes: ALDC - 15; ALEA - V, 1200; ALEANR - VII, 948; ALeCMan - 273; ALEICan - II, 480; ALCyL - 11326; ALGa - V, 15 y ALEPG - 0440.
} 
1986[1980]), cuyas expresiones metafóricas ("el tiempo es oro", "estoy malgastando mi tiempo", "me ha costado tres horas memorizar el examen", etc.) son una muestra de cómo se conceptualizan aspectos de la vida cotidiana mediante estructuras metafóricas y de cómo esto se refleja en el lenguaje. Por ello, en las últimas décadas, destaca la proliferación de investigaciones destinadas al estudio de la lengua desde la perspectiva cognitiva en las que la metáfora y la metonimia se convierten en instrumentos de análisis imprescindibles (Hilferty 1995; Ruiz de Mendoza 1999; Barcelona 2000; Kövecses 2000b; Koch 2001; Llamas 2005).

Los estudios lexicológicos, tanto diacrónicos (Santos y Espinosa 1996; Koch y Blank 1999; Blank 2003; Dworkin 2006a y 2006b) como sincrónicos (Echevarría 2003; Mateu 2009), son un buen ejemplo de la relevancia de estos mecanismos cognitivos. En este sentido, la relación onomasiológica de los datos de los mapas permite que los atlas lingüísticos se conciban como grandes corpus de datos léxicos a partir de cuyo análisis semántico-cognitivo pueden estudiarse con detalle las diferentes estrategias de creación léxica de las que se sirven los hablantes.

\subsection{Geografía lingüística, léxico y semántica cognitiva}

A pesar del considerable aumento del número de investigaciones léxicas en las que se aplican los postulados cognitivos de la metáfora y la metonimia (Clavería en prensa), existen disciplinas en las que no es habitual el empleo de este marco teórico. Una de ellas es la geografía lingüística, una metodología de estudio de la dialectología que nace a finales del siglo XIX y que se caracteriza por representar materiales lingüísticos (fonéticos, fonológicos, morfológicos y léxicos) en mapas geográficos (Iordan 1967: 251-503; Coseriu 1977; García Mouton 1996, 2007, 2010). Aunque los mapas recogen características referidas a distintos aspectos de la lengua, desde la publicación del AIS (Sprach- und Sachatlas Italiens und der Südschweiz) el léxico alcanza una mayor representatividad en los cuestionarios y en las posteriores proyecciones cartográficas que conforman los atlas ${ }^{7}$. Sin embargo, pese a la ingente cantidad de datos primordialmente léxico-semánticos que ofrecen los mapas geolingüísticos, escasean los estudios destinados a analizarlos. En palabras de García Mouton (1990: 26), «parece como si la función de los atlas estuviera cumplida con su edición y fueran obras acabadas en sí mismas, cuando proporcionan material para elaboraciones posteriores».

Una de los primeras investigaciones en las que se pretende dar cuenta de las principales aportaciones que los datos de los atlas pueden suponer en el conjunto de la lingüística se debe a Karl Jaberg (1959[1908]). Con su trabajo, en el que

${ }^{7}$ El mayor interés por el estudio del léxico en los atlas lingüísticos puede verse reflejado, por ejemplo, en los cuestionarios del $A L P I$ y de algunos atlas regionales peninsulares. En el caso del ALEA destaca la abismal diferencia, según datos extraídos de González González (1992:159) entre el número de preguntas destinadas a la fonética, la sintaxis y la morfología (290 preguntas) y las que se dedican al léxico (2.000 preguntas). 
analizaba algunos mapas del $A L F$, se propuso mostrar y ejemplificar «los problemas para los que la geografía lingüística puede encontrar una solución y qué procedimientos sigue para lograr este fin» (Jaberg 1959[1908]:13). En el último capítulo de la obra, mediante el análisis del mapa referido al concepto 'culotte' ( $A L F$ mapa XI - 373), ilustra el cambio semántico de la voz francesa chausses que en algunas zonas (Lorena y Suiza) pasó de designar las antiguas 'medias' a referirse a los 'pantalones'. En palabras del mismo investigador, en este caso, el problema para el que los mapas de la geografía lingüística tienen solución explicaría el modo en el que se comporta la lengua cuando se da un cambio semántico: «una vieja palabra (chausses 'medias') 8 pasa a significar un nuevo concepto (chausses 'pantalones'), pero la vieja idea subsiste (bas 'medias', chaussette 'medias', chausson 'medias')» (Jaberg 1959[1908]: 13).

Asimismo, García Mouton (1990), también con el fin de demostrar la importancia que poseen los datos que pueden aportar los atlas al estudio del léxico, muestra ejemplos de mapas de diversos atlas regionales del español ( $A L E A$, ALEANR, ALEICan) con los que se pueden aclarar ciertas etimologías, establecer la historia de ciertas voces, completar informaciones lexicográficas, etc. En conjunto, a partir del análisis de mapas regionales, la investigadora demuestra la necesidad de dedicar más estudios a los datos de los atlas para la caracterización del léxico hispánico.

El examen de las características etimológico-semánticas de los mapas del párpado contribuirá, en la medida de lo posible y en la línea de Jaberg (1959[1908]) y García Mouton (1990), a demostrar que los atlas constituyen corpus de datos relevantes para llevar a cabo la descripción de las características del léxico de una variedad lingüística. Más concretamente, en la presente investigación, el estudio de las formas de designar el párpado aportará datos sobre los mecanismos que subyacen a los procedimientos de creación léxica de los nombres relativos a partes del cuerpo humano. Con esta finalidad, se ha estructurado el análisis en cuatro apartados distintos en los que se describe la distribución geográfica de las variantes y sus características $(\S 2)$; se analizan los nombres que proceden de las bases latinas PALPĚBRA y PALPĚTRA, -UM ( $(3)$; las formas de origen metafóricometonímico (§ 4); y las designaciones de carácter descriptivo (§5).

${ }^{8}$ Las informaciones que aparecen entre paréntesis son nuestras. 


\section{Variantes léxicas del párpado: características y distribución geográfica ${ }^{9}$}

\subsection{La variación léxica y la prototipicidad}

El párpado es un concepto relevante en los mapas lingüísticos iberorrománicos no sólo porque se halla en el cuestionario de la mayoría de atlas, como se ha mencionado anteriormente $(\S 1)$, sino por el importante grado de variación léxica que presenta en ellos (más de 60 formas léxicas).

En investigaciones previas (Julià 2007, 2009a, 2010), se ha podido advertir que parece que las partes del cuerpo que presentan un mayor grado de variación designativa se caracterizan por ser las menos prototípicas (Rosch 1978; Taylor 1989; Kleiber 1995) entre las categorías asociadas al dominio de partes del cuerpo. Si se identifica la idea de prototipo como el mejor ejemplar de una categoría determinada (Cifuentes Honrubia 1992), el pie y la mano serían miembros corporales prototípicos frente a la pupila o al dedo meñique que quedarían más alejados de este concepto. Así pues, los primeros elementos léxico-semánticos (mano y pie) responderían a las características de un prototipo porque, según Rosch (1978), los mejores ejemplares de una categoría se conceptualizan más rápido, son los primeros que aprenden los niños en relación con la categoría cuerpo humano y sirven de punto de referencia cognitiva.

Consecuentemente, los conceptos de partes del cuerpo que suelen presentar mayor variación denominativa en los atlas, con frecuencia son miembros internos huesos, órganos, músculos - o partes de pequeñas dimensiones que se incluyen en una mayor - las partes del ojo o de la mano, por ejemplo- . De este modo, los mapas sobre conceptos como 'pierna', 'ojos' o 'mano' acostumbran a ser objeto de interés de menos atlas debido a que presentan muy poca o ninguna variación léxica.

Si se interpretan estos datos desde la perspectiva experiencialista y cognitiva, parece que podría relacionarse el grado de variación léxica con el valor que estos conceptos poseen en la conceptualización de la realidad. Por un lado, las partes del cuerpo que suelen presentar un mayor número de variantes designativas y poseen una importante representación en la geografía lingüística son las menos prototípicas, es decir, las más alejadas de la categoría que representaría el dominio de las partes del cuerpo humano. Por otro lado, los miembros corporales sobre los que no se registran variantes denominativas en los atlas lingüísticos suelen coincidir con aquellos que son concepto fuente y que se emplean para comprender multiplicidad de aspectos de la vida cotidiana. Véase, por ejemplo, en el ámbito de la fraseología, como algunas unidades pluriverbales que contienen los lexemas boca (p. e. "abrir la boca", "cerrar la boca", cfr. Olza 2007) y orejas (p. e. "afinar las orejas", "pegar las orejas", cfr. Olza 2009) sirven para conceptualizar las acciones que se

\footnotetext{
${ }^{9}$ Las abreviaturas empleadas para hacer referencia a las variedades lingüísticas son las siguientes: asturleonés (ast.-leon.), catalán (cat.), español (esp.), eusquera (eusq.), francés ( $f r$.), gallego (gall.), italiano (it.), latín (lat.), portugués (port.), rumano (rum.)
} 
realizan con estas partes del cuerpo, hablar y escuchar u oír, respectivamente. En cambio, las partes del cuerpo que presentan un mayor grado de variación léxica parece que son, habitualmente, concepto meta, pues suelen comprenderse, según se deduce del análisis de sus designaciones, a partir de realidades ajenas a ellas con las que guardan cierto parecido formal, estructural o de otro tipo. A modo de ilustración, pueden citarse algunos nombres de la yema del dedo recogidos en el ALEANR (mapa 1477, vol. XI), que surgen de la comparación de esta parte del dedo con otras partes del cuerpo (esp. coronilla y cat. panxeta) o con alguna fruta (esp. manzanilla, cat. mansaneta del dit).

\subsection{Clasificación léxico-semántica y características de las designaciones}

Las designaciones presentan ciertas características que permiten realizar la siguiente clasificación léxico-semántica en tres grupos ${ }^{10}$ :

(a) Denominaciones que proceden de las bases latinas PALPĚBRA y PALPĚTRA, -UM. En este grupo se incluyen las formas procedentes del lat. clás. PALPĚBRA que han llegado a las variedades iberorrománicas bien por transmisión culta (gall. y port. pálpebra ${ }^{11}$ ) bien por transmisión popular (lat. vulg. PALPĚTRUM $>$ esp. párpado y lat. vulg. PALPĚTRA $>$ cat. parpella). En este grupo, se recogen también derivados (esp. parpillo) ${ }^{12}$ y formas complejas que contienen las voces iberorrománicas que tienen como origen las formas latinas mencionadas (esp. párpados de los ojos, párpados de arriba; cat. palpa ${ }^{13}$ de l'ull; port. pálpebra do olho, pálpula da vista) $)^{14}$.

(b) Denominaciones metafóricas o metonímicas. Se trata del conjunto de formas de referirse al párpado que implica una comparación con realidades de otro dominio conceptual o una transferencia metonímica dentro del ámbito léxico-semántico de las partes del ojo.

${ }^{10}$ En la clasificación que se ha realizado, debido a que el estudio se ha centrado en el español, catalán, gallego y portugués, no se han incluido las formas de lenguas no iberorrománicas que se hallan en algunos de los atlas analizados: el fr. paupières (ALDC 9 - Cornellà de Conflent), el it. palpebras (ALDC 85 - Alguer), el eusq. betazalak (ALEANR, Na 200 - Escároz) y el eusq. begigañak ( $A L E A N R$, Na 100 - Vera de Bidasoa). Asimismo, debe mencionarse que ha sido imposible desentrañar el origen de la forma cat. petadors de ses celles (ALDC, 74 - Sóller) y las port. cufela y coufela ( $A L P E G, \mathrm{P} 2$ Gião), motivo por el cual no se han podido recoger en ninguno de los grupos establecidos.

${ }^{11}$ Junto a pálpebra, el $A L E P G$ registra las variantes formales pálpula y paupérola (C 3 - Mira).

${ }^{12}$ Este derivado se ha recogido en una ocasión en el $A L C y L(\mathrm{Bu} 201$ - Berberana).

${ }^{13}$ Palpa es variante formal del catalán parpella (DECat s. v. parpella).

${ }^{14}$ Párpados de los ojos (ALEA, Gr 405 - Gor), párpados de arriba (ALeCMan, Cu 604 - Mira), palpa de l'ull (ALEANR, Hu 404 - Tolva y Hu 408 - Albelda) y pálpebra do olho (ALEPG, L 3 Boca da Mata). 
(b.1.) Español (11): casa del ojo, cobertera del ojo, cubierta del ojo, lo blanco del ojo, clara del ojo, mejillas del ojo, pestañas, ribetes del ojo, tapas del ojo, tela y tela del ojo. ${ }^{15}$

(b.2.) Catalán (13): celles, clotxa de s'ull, cim de l'ull, guarnició de l'ull, olla de s'ull, pestanyes, palpanyes, pellofa de s'ull, tanca, tapa, tapes de l'ull, tapetes de l'ull y tapa des párpados. ${ }^{16}$

(b.3.) Gallego (8): capel, capela, capelo do ollo, conca, conca do ollo, perceba ${ }^{17}$, pestana (pestaña $)^{18}$ y tapa do ollo. ${ }^{19}$

(b.4.) Portugués (13): barbatana, capa, capa da vista, capa do olho, capela, capela do olho, capela da vista, capulho do olho, carapela do olho, carapulinha, celha, cobertora y tampa da vista. ${ }^{20}$

\footnotetext{
${ }^{15}$ Casa del ojo (ALeCMan, Ab 307 - El Bonillo), cobertera del ojo (ALEA, Gr 301 - Colomera), cubierta del ojo (ALEA, Gr 200 - La Puebla de don Fadrique), clara del ojo (ALEICan, GC 11 Artenara), lo blanco del ojo (ALEICan, GC 4 - San Bartolomé de Tirajana, 20 - Teror, 40 - Agüimes); mejillas del ojo (ALEANR, Gu 200 - Tortuera), pestañas (ALEA, Co 302 - Palma del Río; ALEANR, Hu - Lasieso, 206 - Aínsa; Te 100 - Nogueras; Lo 501 - Viniegra de Arriba; ALeCMan, Ab 211 - Motilleja), ribetes del ojo (ALEA, Gr 304 - Diezma), tapas del ojo (ALEA, J 400 - Santiago de la Espada), tela (ALEANR, Na 106 - Ollo) y tela del ojo (ALEA, Gr 302 - Iznalloz).

${ }^{16}$ Celles (ALDC, 43 - Llagostera), clotxa de l'ull (ALDC, 118 - Fraga), cim de l'ull (ALDC, 26 Lledó d'Empordà), guarnició de l'ull (ALDC, 90 - Taüll), olla de s'ull (ALDC, 84 - Eivissa), palpanyes (86 - Esterri d'À̀neu), pellofa de s'ull (ALDC, 83 - Sant Josep de Sa Talaia), pestanyes (ALDC, 23 - Oix, 26 - Lledó d'Empordà, 30 - Les Llosses, 31 - Banyoles, 34 - Santa María de Corcó, 38 - La Ginebrosa, 48 - Castellfollit de Riubergós, 53 - Freixenet de Segarra, 59 - Sant Jaume de Sesoliveres, 69 - Cambrils de Mar, 87 - Cerler, 88 - Ordino, 89 - Senet de Barravés, 97 - Arfa, 110 - Cubells, 111 - Almenar, 113 - L'Ametlla de Mar, 115 - Saidí), tanca (ALDC, 123 - Margalef de Montsant), tapa (ALDC, 71 - Es Migjorn Gran), tapes de l'ull (ALDC, 168 - L'Alcúdia de Carlet, 169 - Albalat de Ribera, 171 - Alberic, 173 - Simat de Valldigna, 181 - Muro del Comtat, 182 - Tàrbena), tapetes de l'ull (ALDC, 40 - Llobera de Solsonès) y tapa des párpados (ALDC, 170 - Alzira).

${ }^{17}$ Perceba es variante del gallego perfeba 'pestaña' (cfr. $§ 3.2$. y $\S 4.2$ ).

${ }^{18}$ En dos puntos de encuesta del $A L G a$, se ha recogido la forma castellanizada pestaña (P 16 Sanxenxo; O 31 - Lobios).

${ }^{19}$ Capel ( $A L G a, \mathrm{C} 40$ - Touro), capela (ALGa, P 8 - Rodeiro, P 11 - Dozón, C 32 - Toques, O 26 - Entrimo, L 28 - Palas de Rei), capelo do ollo ( $A L G a$, O 2 - Boborás), conca (ALGa, L 19 - Nogueira de Muñiz), conca do ollo (ALGa, L 13 - A Pontenova, 16 - Consagrada), perceba ( $A L G a$, L 20 Friol), pestana ( $A L G a, \mathrm{C} 12$ - Malpica de Bergantiños, 20 - Aranga, 21 - Camariñas, 22 - Vimianzo, 23 - Cerceda, 37 - Carnota; P 20 - O Covelo, 29 - Salceda de Caselas; Le 2- Vilafranca do Bierzo), papo do ollo ( $A L G a$, C 16 - Ponteceso) y tapa do ollo ( $A L G a$, P 31 - Oia, 33 - A Guarda; O 31 Lobios).

${ }^{20}$ Barbatana (ALEPG, Aç 3 - Ponta Ruiva), capela do olho (ALEPG, Aç 12 - Frontinhas), capulho do olho (ALEPG, Bç 3 - Constantim), carapela do olho (ALEPG, Bç 8 - Penas Roias), carapulinha (ALEPG, V 8 - Granja do Tedo), cobertora (VC 4 - Estrica), celha (ALEPG, Bç 2 - Guadramil) y tampa da vista ( $A L E P G, \mathrm{~F} 11$ - Alta Mora). Existe un grupo de formas que no se pueden situar geográficamente con exactitud porque en el mapa aparecen representadas con el mismo icono. Se trata de las designaciones capa, capa da vista, capa do olho, capela y capela da vista cuyo símbolo de representación es un triángulo de color azul para todos los casos.
} 
(c) Denominaciones descriptivas. En este grupo designativo se han agrupado las formas de referirse al párpado que surgen de la descripción de algunas de sus características físicas: es una membrana que está recubierta de piel (piel, pell, papo) y se ubica en el ojo (del ojo, de la bola del ojo).

(c.1.) Español (4): piel del ojo; pellejo del ojo, pellejo de la bola del ojo y pellejo del párpado. ${ }^{21}$

(c.2.) Catalán (3): pell, pell de l'ull y pell de les pestañes. ${ }^{22}$

(c.3.) Gallego (3): papo do ollo, piel do ollo y sobreollo. ${ }^{23}$

(c.4.) Portugués (4): papo, papo do olho, pele y pele da vista. ${ }^{24}$

Un importante número de las variantes, aunque desde el punto de vista etimológico y motivacional son distintas, comparte dos características que conviene tener en cuenta en el estudio semántico: son respuestas únicas y lexías complejas.

Muchas de las formas, principalmente las de origen metafórico, constituyen respuestas únicas en los mapas, pues se recogen en una ocasión en un solo punto de encuesta. Este aspecto, aunque desde la perspectiva geolingüística podría hacer creer que se trata de respuestas aisladas y de poca importancia en el conjunto de los datos estudiados, resulta muy interesante semánticamente, como se pudo comprobar en el análisis de los nombres de la pupila (Julià 2009a) y en el de los nombres de los dedos de la mano (Julià 2010). El examen semántico de respuestas únicas como señorita 'pupila' o el dedo de la arteria 'dedo corazón',25, entre otros muchos ejemplos, permite advertir la existencia de un conjunto de procedimientos de conceptualización y designación comunes a las variedades iberorrománicas.

La mayoría de las designaciones son lexías complejas (esp. casa del ojo, cat. guarnició de l'ull, gall. conca do ollo, port. tampa da vista) que, tal y como se demostró en Julià (2009a: 108-111) para algunos nombres de la pupila (esp. niña del ojo, luna del ojo; cat. animeta de l'ull, nineta de l'ull), no deben considerarse compuestos sintagmáticos a pesar de que cumplen muchos de los requisitos que permitirían clasificarlos como tales (Bustos Gisbert 1986; Val Álvaro 1999: 4824; Buenafuentes 2007 y 2010). El sintagma preposicional de estas formaciones -

${ }^{21}$ Piel del ojo (ALEA, Gr 410 - Dehesa de Guadix), pellejo del ojo (ALEA, Al 201 - Vélez Rubio; Gr 403 - Pedro Martínez, 604 - Albuño), pellejo de la bola del ojo (ALEA, J 404 - Larva) y pellejo del párpado (ALEA, $\mathrm{H} 600$ - Beas).

${ }^{22}$ Pell (ALDC, 56 - Sant Andreu de Llavaneres, 69 - Cambrils de Mar, 73 - Pollença, 89 - Senet de Barravés, 98 - Sarroca de Bellera, 187 - El Pinós de Monòver), pell de les pestanyes (ALDC, 18 Vilallonga de Ter y 149 - Vilafranca del Maestrat) y pell de l'ull (ALDC, 78 - Montuïri, 104 - Isona, 149 - Vilafranca del Maestrat).

${ }^{23}$ Piel do ollo (ALGa, en C 31 - Oroso) y sobreollo ( $A L G a$, P 16 - Sanxenxo).

${ }^{24}$ Papo (ALEPG, M 4 - Estreito de Câmara de Lobos, M 6 - Boaventura), papo do olho (ALEPG, M 5 - Boaventura), pele (ALEPG, P 3 - Sardoura (Aveiro)) y pele da vista (ALEPG, VC 7 - Castelo de Neiva).

${ }^{25}$ Señorita 'pupila' (ALEANR, mapa 949, vol. VII; Te 308 - Teruel); el dedo de la arteria (ALEA, mapa 1272, vol. V; Ma 200- Alameda). 
siempre referido al ojo (esp. mejillas del ojo), a una de sus partes (cat. tapa des párpados, petadors de ses celles) o al sentido de la vista (port. tampa da vista) posee un carácter meramente referencial y carece de valor semántico. Esta característica del sintagma preposicional deriva, muy probablemente, de la metodología de recogida de datos de la geografía lingüística (González González 1992) ya que las designaciones obtenidas surgen como respuesta a una pregunta aislada. En el caso del $A L E A$, por ejemplo, en el mapa se menciona que para obtener la forma con la que los informantes se referían al párpado señalaban esta parte del cuerpo con su dedo. La situación comunicativa creada por el encuestador, por tanto, favorece la aparición del elemento referencial del compuesto, puesto que con él se ubica la denominación que se está emitiendo. Además, son numerosos los casos en los que los atlas atestiguan el primer sustantivo de la lexía compleja sin el sintagma preposicional (p. e. cat. tanca $\sim$ tanca de l'ull; gall. conca $\sim$ conca do ollo; port. capa capa da vista). La prescindibilidad de la segunda parte de la lexía compleja es lo que permite confirmar que no se pueden clasificar estas formas como compuestos sintagmáticos porque carecen de la unidad de imagen que caracteriza a estas formaciones (p. e. diente de león 'hierba' *diente 'hierba'), esto es, no mantienen la relación biunívoca que, desde un punto de vista semántico, se debería establecer entre los dos elementos (Bustos Gisbert 1986: 94-95).

\subsection{Distribución geográfica de las variantes}

La distribución geográfica de las variantes y sus características léxicosemánticas (§ 2.2.) tienen la siguiente representación cartográfica:

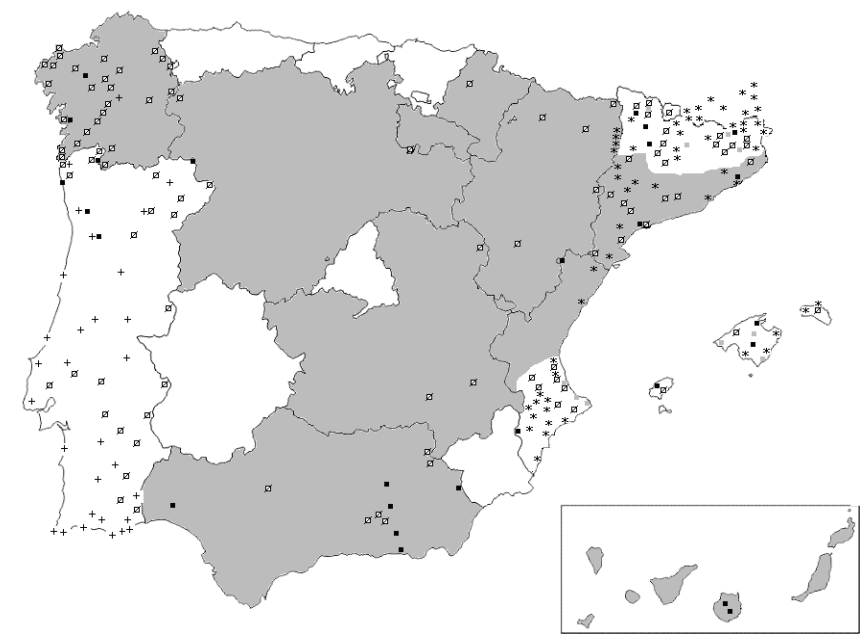

$$
\begin{aligned}
& \text { mpárpado Ø denominaciones metafóricas } \mathrm{y} / 0 \text { metonimicas } \\
& { }^{*} \text { parpella } \\
& + \text { pálpebra denominaciones descriptivas }
\end{aligned}
$$




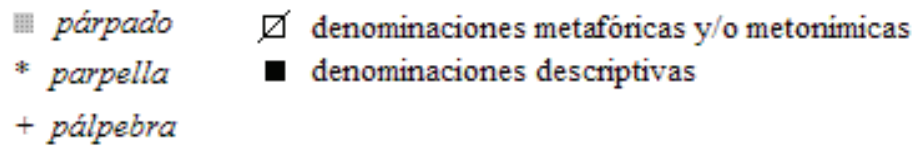

Mapa 1. Denominaciones iberorrománicas del párpado ${ }^{26}$

Las formas de uso más frecuente para referirse al párpado en las variedades iberorrománicas son las que proceden del la base etimológica latina PALPĚBRA. Tanto la designación patrimonial española (párpado) como la catalana (parpella), presentan un importante número de variantes fonético-fonológicas: párpado (párparos, párpagos, párpanos, párpalos, pálparos, pálpados, pálpagos ${ }^{27}$ y parpella (parpalls, pipelles, perpalles, palpellas, palpes, parpes, pàrpols, pàmpols, parpos, pàrpares, pàrpars, parps, parpus, parcs). Este elevado número de variantes formales, al menos en español, se debe a que la palabra posee una estructura fonética marcada, pues contiene dos vocales centrales (párpado) y tres consonantes oclusivas (párpado), lo que facilita que, junto a los procesos evolutivos de tipo regular, se produzcan otros de asimilación y disimilación irregulares. Por tanto, la variación formal está condicionada por las características fonéticas de la voz.

En el caso del español, de todas las variantes recogidas en los mapas, las más extendidas son párpago, párpalo y párparo. En el $D E C H$ (s. v. párpado), se hace referencia al carácter dialectal de estas variantes fonético-fonológicas. Párpago se identifica como voz propia «de Andalucía, Cespedosa y seguramente otros lugares», el uso de párpalo se sitúa en la Sierra de Gata y el de la forma párparo se ubica en Galicia, en Chile, Perú, Colombia, Venezuela, América Central, Méjico y también en el judeoespañol. Las informaciones de los atlas permiten complementar los testimonios dialectales que aporta el $\mathrm{DECH}^{28}$ en lo que respecta a la extensión del uso de las distintas formas de párpado. Párpago parece ser la

${ }^{26}$ Las respuestas concernientes a las islas Azores y Madeira del $A L E P G$ y a la isla de Cerdeña del $A L D C$ se han tenido en cuenta para la investigación pero no se han representado cartográficamente. Asimismo, para los territorios del mapa que aparecen totalmente en blanco, no se han analizado datos o bien porque no existen atlas de la zona (Asturias, Extremadura, Madrid, Murcia) o bien porque los atlas de la región no contienen la cuestión referente al párpado (ALECant, Cantabria) o porque no pertenecen a una zona de habla exclusivamente románica (País Vasco).

${ }^{27}$ Distribución de las variantes según el atlas en el que se encuentran: ALEA (párparos, párpagos, párpalos), ALEANR (pálparos, párpalos, párpagos, pálpados, pálpagos), ALECant (párpagos), ALECMan (pálpagos, párpalos, párpagos), ALCyL (párparos, párpalos, párpagos), ALEICan (párparos, párpagos), ALGa (pálparos, párparos, párpalos, pálpados, párpanos) y ALDC (pálpados, párpalos, párpros, párpagos).

${ }^{28}$ Sobre el valor complementario de los datos de los atlas al $D E C H$, véase Prat (2006). 
variante más empleada en español, pues se recoge en multiplicidad de puntos de encuesta de Andalucía, las Islas Canarias, Aragón, Castilla y León y Castilla La Mancha. En algunos de estos territorios (Andalucía ${ }^{29}$ y Canarias), su uso es más habitual que el de la forma estándar (párpado) y, en otros (Aragón y Castilla La Mancha), párpado y párpago poseen una frecuencia de uso similar.

En el dominio lingüístico catalán, como se refleja en el mapa 1, se advierte que existen más variantes denominativas que en español. Además de existir más ejemplos de la denominación española (párpados) y de sus variantes más extendidas (párparos, párpalos, párpagos ${ }^{30}$ ) en buena parte del territorio, el uso del catalán estándar parpella — ubicado principalmente en la zona francesa de habla catalana, en algunas localidades de Gerona y en el punto de encuesta del Alghero (Cerdeña) - convive con un importante número de formas de origen metafóricometonímico (§ 4) y descriptivo (§5). Asimismo, debe destacarse el conjunto de variantes formales de parpella sobre las que el DECat (s. v. parpella) y el DCVB (s. v. parpella) aportan informaciones históricas y diatópicas. Parpalls y pipelles son, quizá, las variantes más significativas, pues constituyen las denominaciones propias del valenciano y del catalán hablado en las Islas Baleares, respectivamente, según datos de las dos obras lexicográficas mencionadas. En el DECat se indica que ambas variantes, igual que el estándar y moderno parpella, se documentan por primera vez en el siglo XIX (parpall en 1800; pipella principios de siglo; parpella en 1864). El resto de formas podrían calificarse, según el mismo diccionario, como variantes locales. Pàrpols se documenta en el siglo XVI (DECat) y casi todos sus ejemplos de uso del $A L D C$ se sitúan en el sur de Alicante, aunque también es posible hallar casos en puntos aislados de Lleida, Tarragona y Castellón. Destaca, por su supuesto origen motivacional, la variante formal pàmpols ${ }^{31}$. Es probable que se deba a un proceso de homonimización semántica (Veny 1991: 83) ya que esta voz posee una evidente semejanza formal con pàrpols. Así, el uso de pàmpols por pàrpols surgiría de la interferencia formal que provoca el contenido semántico del parónimo pàmpols — que suele emplearse para designar realidades distintas al párpado ('hoja de la vid', 'pantalla de las lámparas', un tipo de 'pez' $)^{32}$ - sobre pàrpols. Concretamente, el significado que parece provocar el uso de pàmpols para designar el párpado es el de 'pantalla de una lámpara'. Si fuera de este modo, esta designación procedería de un proceso semántico metafó-

${ }^{29}$ El genuino párpado únicamente se ha hallado en 7 de los 230 puntos de encuesta del ALEA (J 201 - Orcera; Al 405 - Gafarillos, Al 600 - Carboneras; Ma 406 - Málaga; Se 307 - Sevilla; H 401 Campofrío, H 502 - Moguer).

${ }^{30} \mathrm{Se}$ ha considerado que la forma pagus, recogida en una única ocasión (57 - Aguiló), es acortamiento de la variante española párpagos.

${ }^{31}$ Pàmpols se halla en el punto 114 (Cervera) y los únicos ejemplos de pàrpols de la comunidad catalana pertenecen al punto 113 (Linyola) y al 117 (Arbeca), el resto de formas se ubican, principalmente, en la zona de Alicante.

${ }^{32}$ La información semántica se ha extraído del DIEC (s. v. pàmpol). 
rico a partir de la comparación del párpado con una tapa u objeto que sirve para hacer de pantalla, mampara o cubierta, lo que permitiría vincular esta designación con el resto de formas estudiadas que se identifican con una METÁFORA DE RECIPIENTE (§ 4.1.1.). Igualmente, deben distinguirse en un grupo las denominaciones palpes, parpes y palpa de l'ull, localizadas en la zona de la frontera catalanaaragonesa, porque parecen las variantes formalmente más próximas al catalán antiguo pàlpet (1. ${ }^{\text {a }}$ doc. segunda mitad del siglo XIV). Estas podrían estar relacionadas con las designaciones pàlpet (Tremp) y pàrpat (Cadaqués) que Coromines (DECat) define como arcaísmos. Las demás variantes formales halladas en el ALDC (perpalles, palpellas, parpos, pàrpares, parps, parcs) constituyen respuestas únicas, pues se recogen en un solo punto de encuesta ${ }^{33}$.

Como refleja el mapa 1, se puede advertir que las designaciones que se originan mediante mecanismos semánticos metafórico-metonímicos - representados mediante un icono que se corresponde con un cuadro blanco y una línea diagonal en el mapa- constituyen el segundo conjunto denominativo más usual para referirse al párpado en las variedades iberorrománicas (vid. § 4). Asimismo, se deduce también que, en los dominios lingüísticos del gallego, portugués y catalán, las formas surgidas por estos procesos son más habituales que en español, variedad en la que, según las informaciones del mapa, son escasas las designaciones ajenas a la forma patrimonial párpado.

Finalmente, los datos del mapa revelan que las formas de carácter descriptivo, aquellas que surgen de la simple observación de las particularidades del concepto designado por el hablante y que se representan en el mapa con un cuadro de color negro, constituyen un reducido grupo de denominaciones cuya ubicación se limita a algunos puntos de encuesta del dominio lingüístico gallego-portugués, del catalán y a unas pocas localidades andaluzas y canarias. Las características sintáctico-semánticas de este conjunto denominativo se analizan en el epígrafe quinto del artículo $(\S 5)$.

\section{Bibliografía}

(a) Atlas lingüísticos

[AIS] JABERG, Karl y Jakob JUD (1928-1940): Sprach-und Sachatlas Italiens und der Südschweiz. Zofingen: Gedruck mit Unterstützung der Gesellschaft für

${ }^{33}$ Perpalles (154 - Castelló de la Plana), palpellas (85 - Alguer), parpos (117 - Arbeca), pàrpares (63 - Sant Boi del Llobregat), parps (1. ${ }^{\mathrm{a}}$ resp. 24 - Castellar de N'Hug), parcs (2. ${ }^{\mathrm{a}}$ resp. 24 Castellar de N'Hug). 
Wissenschaftliche Forschung an der Universität Zurich und privater Freunde des Werkes von der Verlagsanstalt Ringier \& Co.

[ALAnd] GRIERA, Antoni (1973): Atles Lingüistic d'Andorra. Barcelona: Polígrafa.

[ALCyL] AlVAR, Manuel (1999): Atlas lingüistico de Castilla y León. Salamanca: Junta de Castilla y León-Consejería de Educación y Cultura, 3 vols.

[ALDC] VENY CLAR, Joan y Lídia PONS GRIERA (2001-): Atles lingüístic del domini català. Barcelona: Institut d'Estudis Catalans.

[ALEA] ALVAR, Manuel (1963-1973): Atlas lingüistico y etnográfico de Andalucía. Granada: Universidad de Granada, 6 vols.

[ALEANR] ALVAR, Manuel (1979-1983): Atlas lingüístico y etnográfico de Aragón, Navarra y Rioja (con la colaboración de Antonio Llorente, Tomás Buesa y Elena Alvar). Madrid: La Muralla, 12 vols.

[ALEICan] ALVAR, Manuel (1975-1978): Atlas lingüístico y etnográfico de las Islas Canarias. Madrid: La Muralla, 3 vols.

[ALECant] ALVAR, Manuel (1995): Atlas lingüistico y etnográfico de Cantabria. Madrid: Arco/Libros, 2 vols.

[ALeCMan] García Mouton, Pilar y Francisco Moreno Fernández (1987-): Atlas lingüístico y etnográfico de Castilla La Mancha. [En línea, $<$ http://www.uah.es/otrosweb/ alecman/>].

[ALEPG] SARAMAGO, João (dir.) (en prensa): Atlas Linguístico-Etnográfico da Portugal e da Galiza. Lisboa: Centro de Linguística da Universidade de Lisboa.

[ALF] GiLliÉRON, Jules y Edmond EdMONT (1902-1910): Atlas Linguistique de la France. Paris: Honoré Champion, 12 vols.

[ALGa] García GonZÁlez, Constantino y Antón SANTAMarina Delgado (1990-): Atlas lingüistico galego. Santiago de Compostela: Universidade de Santiago-Instituto da Lingua Galega, 6 vols.

(b) Referencias bibliográficas

ALINEI, Mario (1984): Dal totemismo al cristianesimo popolare. Sviluppi nei dialetti italiani ed europei. Torino: Edizioni dell'Orso.

- (1989): "Geografia semantica: continuatori di draco in Italia e in Francia", in Études de dialectologie et de géolinguistique offerts à Gaston Tuaillon, vol. II, pp. 459-487. Grenoble: Ellug/Université Stendhal Grenoble 3.

- (1997) : "L'aspect magico-religieux dans la zoonymie populaire", in Les zoonymes. Actes du colloque international tenu à Nice les 23, 24, 25 janvier 1997, Sylvie Mellet (ed.), pp. 9-22. Nice: Centre de recherches comparatives sur les langues de la Méditerranée ancienne. 
- (2002): "Il ruolo della motivazione nel lessico", in Dialectoloxía e Léxico, Rosario Álvarez Blanco, Francisco Dubert García y Xulio Sousa Fernández (eds.), pp. 15-28. Santiago de Compostela: Consello da Cultura Galega/Instituto da Lingua Galega.

AlVAR, Manuel (1982): “Atlas lingüísticos y diccionarios”. Lingüistica Española Actual IV/2: 253-323.

Álvarez PÉrez, Xosé A. (2009): "Herramientas informáticas para el estudio diacrónico del gallego", in Tendencias actuales en la investigación diacrónica de la lengua, Laura Romero Aguilera y Carolina Julià Luna (coords.), pp. 117124. Barcelona: Publicacions i Edicions de la Universitat de Barcelona.

ANDRE, Jacques (1978): Les mots a redoublement en latin. Paris: Éditions Klincksieck.

- (1991): Le vocabulaire latin de l'anatomie. Paris: Les Belles Lettres.

BuENAFUENTES DE LA MATA, Cristina (2007): Procesos de gramaticalización y lexicalización en la formación de compuestos en español, 2 vols. Bellaterra: Universitat Autònoma de Barcelona. [Tesis doctoral digitalizada en $<$ http://www.tesisenxarxa.net/TDX-0321107-17284>].

- (2010): La composición sintagmática en español. San Millán de la Cogolla: Cilengua.

BROwn, Cecil H. y Stanley R. WITKOWSKI (1981): "Figurative Language in a Universalist Perspective". American Ethnologist 8/3: 596-615.

Bustos GisBerT, Eugenio de (1986): La composición nominal en español. Salamanca: Ediciones Universidad de Salamanca.

CAPRINI, Rita y Rosa RONZITTI (2007): "Studio iconomastico dei nomi della 'pupilla' nelle lingue indoeuropee e nei dialetti romanzi". Quaderni di Semantica XXVIII/2: 287-326.

CARPITELLI, Elisabetta (2006): "Il nome della trottola in Alta Val di Magra: fra onomasiologia e semasiologia". Quaderni di Semantica XVII/1-2: 167-181.

CASTAÑER MARTÍN, Rosa M. a (2009): "El atlas lingüístico como fuente lexicográfica. Del ALEANR al DDEAR". Archivo de Filología Aragonesa 65: 139-164.

CifUENTES HonRUBiA, José Luis (1992): "Teoría de prototipos y funcionalidad semántica". Estudios de Lingüistica. Universidad de Alicante (E.L.U.A.) 8: 133-177.

ClAVERÍA NADAL, Gloria (en prensa): "Nuevas perspectivas en el estudio de la evolución del léxico", in Historia del léxico: perspectivas de investigación, Gloria Clavería Nadal, Margarita Freixas Alás, Marta Prat Sabater y Joan Torruella Casañas (eds.). Madrid/Frankfurt: Iberoamericana/Vervuert.

COLÓN DOMÉNECH, Germà (1997): "Entorn l'atles lingüístic d'Andorra", in Estudis de filologia catalana i romànica, pp. 305-327. València-Barcelona: Institut Universitari de Filologia Valenciana-Publicacions de l'Abadia de Montserrat.

CONTINI, Michel (2005): "Formazione fonosimboliche negli zoonimi dell'area romanza. Reflessioni sulle carte dell'AliR", in Els mètodes en dialectologia: 
continuïtat o alternativa. I Jornada de l'Associació d'amics del professor Antoni M. Badia i Margarit (Barcelona, 11 de març de 2004), pp. 67-90. Barcelona: Institut d'Estudis Catalans.

COSERIU, Eugenio (1977): "La geografía lingüística", in El hombre y su lenguaje. Estudios de teoría y metodología lingüistica, pp. 103-158. Madrid: Gredos.

CUENCA ORDIÑANA, M. ${ }^{a}$ Josep y Joseph HILFERTY (1999): Introducción a la lingüistica cognitiva. Barcelona: Ariel.

DALBERA, Jean-Philippe (2006): Des dialectes au langage. Une archéologie du sens. Paris: Honoré Champion.

[DCVB] Alcover i Sureda, Antoni M. ${ }^{a}$ y Francesc De B. Moll i CaSAnovas (2002[1962]): Diccionari català-valencià-balear. Barcelona: Institut d'Estudis Catalans.

[DECat] COROMINAS VigneauX, Joan (1980-1991): Diccionari etimologic i complementari de la llengua catalana (con la colaboración de Joseph Gulsoy y Max Cahner). Barcelona: Curial.

[DEILE] ROBERTS, Edward A. y Bárbara PASTOR (1996): Diccionario etimológico indoeuropeo de la lengua española. Madrid: Alianza.

[DELL] ERNOUT, Alfred y Antoine MEILlET (1967[1932]): Dictionnaire étymologique de la langue latine. Histoire des mots. Paris: Klincksieck.

[DHLF] REY, Alain (dir.) (1992): Dictionnaire historique de la langue française. Paris: Dictionnaires Le Robert.

DÍAZ RoJO, José Antonio (2002): "El fonosimbolismo: ¿propiedad natural o convención cultural?". Revista electrónica de estudios filológicos 3. [En línea, $<$ http://www.um.es/ tonosdigital/znum3/estudios/fonosimbDiazRojo.htm\#_ftn7>].

[DIEC] INSTITUT D'ESTUDIS CATALANS (2007): Diccionari de la llengua catala$n a, 2{ }^{a}$ edición. Barcelona: Edicions 62-Enciclopèdia Catalana. [En línea $<$ http://dlc.iec.cat/ $>$ ].

[DRAG] Real ACADEMIa Galega (1997): Diccionario da Real Academia Galega. A Coruña: Real Academia Galega. [En línea $<$ http://www.edu.xunta.es/diccionarios/index_rag.html >].

DWORKIN, Steven N. (2006a): "La naturaleza del cambio léxico", in Actas del VI Congreso Internacional de Historia de la Lengua Española. Madrid (29 de septiembre a 3 de octubre de 2003), José J. de Bustos Tovar y José L. Girón Alconchel (eds.), vol. I, pp. 67-84. Madrid: Arco/Libros.

- (2006b): "Recent Developments in Spanish (and Romance) Historical Semantics", in Selected Proceedings of the 8th Hispanic Linguistics Symposium, Timothy L. Face y Carol A. Klee (eds.), pp. 50-57. Somerville, MA: Cascadilla Proceedings Project.

Fuster BERENGUER, M. ${ }^{a}$ Trinidad (1996-1997): "Voces de creación metafórica sobre el maíz y el trigo en el Atlas Lingüístico de Aragón, Navarra y La Rioja". Estudios de Lingüística 11: 139-148. 
GARCÍA Mouton, Pilar (1990): "El estudio léxico en los mapas lingüísticos", in Estudios sobre variación lingüística, Francisco Moreno Fernández (recop.), pp. 27-75. Salamanca: Universidad de Alcalá de Henares.

- (1996): "Dialectología y geografía lingüística", in Manual de dialectología hispánica. El español de España, Manuel Alvar (dir.), pp. 63-77. Barcelona: Ariel.

- (2007): "Las disciplinas tradicionales (II). Dialectología y geolingüística", in Manual de lingüística románica, José Enrique Gargallo Gil y María Reina Bastardas (coords.), pp. 319-350. Barcelona: Ariel.

- (2010): "Si el DRAE utilizase los atlas lingüísticos...: un ejemplo aragonés", in De moneda nunca usada. Estudios filológicos dedicados a José M. ${ }^{a}$ Enguita Utrilla, Rosa M. ${ }^{a}$ Castañer Martín y Vicente Lagüéns Gracia (eds.), pp. 271282. Zaragoza: Inst. "Fernando el Católico".

GoOSSENS, Louis (1995): "Metaphtonymy: the Interaction of Metaphor and Metonymy in Figurative Expressions for Linguistic Action", in By Word of Mouth. Metaphor, Metonymy and Linguistic Action in a Cognitive Perspective, Louis Goossens et al. (eds.), pp. 159-174. Amsterdam-Philadelphia: John Benjamins.

GONZÁLEZ GONZÁLEZ, Manuel (1992): "Metodología de los atlas lingüísticos en España", in Nazioarteko Dialektologia Bilzarra. Agiriak. (IKER 7), pp. 151177. Bilbao: Real Academia de la lengua vasca.

Gordón Peral, M. a Dolores y Stefan RUhSTALler KuHNe (2008): “Atlas lingüísticos y toponimia como fuentes en el estudio histórico del léxico", in $A c$ tas del VII Congreso Internacional de Historia de la Lengua Española: Mérida (Yucatán) 4-8 de septiembre de 2006, Concepción Company Company y José G. Moreno de Alba (coords.), vol. II, pp. 1347-1358. Madrid: Arco/Libros.

GoschleR, Juliana (2005): "Embodiment and Body Metaphors". Metaphorik.de 09: 33-52.

HeInE, Bernd (1997): Cognitive Foundations of Grammar. Oxford: Oxford University Press.

[Houaiss] HouAISS, Antônio; Mauro DE SAlles VILlaR y Francisco Manoel DE Mello FrANCO (eds.) (2003): Diccionario Houaiss da língua portuguesa. Lisboa: Temas e Debates.

InTITUTO DA Lingua GALEGa [en línea]: Índices do Atlas lingüístico galego, $<$ http://ilg.usc.es/indices/>. [Consulta: 04/11/2010]

IORDAN, Iorgu (1967): Lingüistica románica. Evolución-corrientes-métodos. Madrid: Ediciones Alcalá [Reelaboración y notas de Manuel Alvar].

JABERG, Karl (1959[1908]): Geografía lingüistica. Ensayo de interpretación del "Atlas Lingüistico de Francia». Granada: Universidad de Granada. [Traducción por Antonio Llorente y Manuel Alvar de Sprachgeographie. Beitrag zum Verstädnis des "Atlas Linguistique de la France». Aurau: Druck und Verlag von H. R. Sauerländer \& Co.] 
JOHNSON, Mark (1992[1987]): El cuerpo en la mente: fundamentos corporales del significado, la imaginación y la razón. Madrid: Debate [Traducción de The Body in the Mind: The Bodily Basis of Meaning, Imagination and Reason. Chicago: The University of Chicago Press].

JULIÀ LUNA, Carolina (2007): Léxico y variación: las denominaciones de las partes del ojo. Bellaterra: Universitat Autònoma de Barcelona. [Trabajo de investigación disponible en línea $<$ http:// www.recercat.net/handle/2072/4360 $>$ ].

- (2009a): "Los nombres de la pupila en los atlas regionales de la Península Ibérica". Lingüistica Española Actual 31/1: 89-131.

- (2009b): "El cuerpo humano en la creación y motivación de los nombres románicos de insectos". Revue de Linguistique Romane tomo 73, n. ${ }^{\circ}$ 291-292: 321 369.

- (2010): Estructura y variación en el léxico del cuerpo humano. Bellaterra: Univeristat Autònoma de Barcelona. [Tesis doctoral dirigida por la Dra. Gloria Clavería Nadal disponible en línea <http://www.tesisenxarxa.net/TDX1222110-163719/>].

KLEIBER, Georges (1995): La semántica de los prototipos: categoría y sentido léxico. Madrid: Visor.

LABOV, William (1974): "The Boundaries of Words and their Meanings", in New Ways of Analyzing Variation in English, Charles J. Baley y Roger W. Shuy (coords.), pp. 340-373. Washington: Georgetown University Press.

LAKOFF, George (1987): "Image Metaphors". Metaphor and Symbolic Activity 2/3: 219-222.

LAKOFF, George, y Mark JOHNSON (1986[1980]): Metáforas de la vida cotidiana. Madrid: Cátedra [Traducción de Metaphors We Live By. Chicago: The University of Chicago Press].

LAKOFF, George, y Mark TURNER (1989): More than Cool Reason: A Field Guide to Poetic Metaphor. Chicago: University of Chicago Press.

Llamas SAíz, Carmen (2005): Metáfora y creación léxica. Pamplona: Ediciones Universidad de Navarra, S. A.

MALKIEL, Yakov (1990): Diachronic Problems on Phonosymbolism. Amsterdam: John Benjamins.

- (1994): "Regular Sound Development Phonosymbolic Orchestration, Disambiguation of Homonyms", in Sound Symbolism, John J. Ohala, Leanne Hinton y Johanna Nichols (eds.), pp. 207-221. Cambridge: Cambridge University Press.

MÁRQUEZ LINARES, Carlos Francisco (1998): La polisemia en el campo léxico "el cuerpo humano": un estudio contrastivo inglés-español, Córdoba: Universidad de Córdoba [Tesis doctoral inédita].

MARTíN-MuniCIO, Ángel (1992): "La metáfora en el lenguaje científico". Boletín de la Real Academia Española LXXII, cuaderno CCLVI: 221-249. 
MARTINS-BALTAR, Michel y Geneviève CALBRIS (1997): Le corps dans la langue. Esquisse d'un dictionnaire onomasiologique. Notions et expressions dans le champ de «dent» et de «manger». Tübingen: Max Niemeyer.

- (2009): "Sémiologie de l'acte 'manger': Source et objet de figures". Semiotica 93/3-4: 207-240.

Mateu Fontanals, Jaume (2009): "Modelos cognitivos", in Panorama de la lexicología, Elena de Miguel (ed.), pp. 281-300. Barcelona: Ariel.

NAVARRO CARRASCO, Ana Isabel (1988): "Voces de creación metafórica en el ALEA". Lingüistica Española Actual X: 107-114.

- (2001): "Yayo en los diccionarios y en los atlas lingüísticos". Revista de Filología Española LXXXI/1-2: 185-193.

NeBRIJA, Antonio de (1973[1516]): Vocabulario de romance en latín. Madrid: Castalia [Introducción de Gerald J. Macdonald].

Negro Romero, Marta (2008): "O léxico tradicional no campo semántico das partes da cabeza: proposta de recuperación nos diccionarios normativos". Estudos de Lingüistica Galega 1: 235-246.

OlZA MORENO, Inés (2007): “¿Cómo conceptualizan el lenguaje los hablantes del español? El caso de los somatismos basados en boca", in Interculturalidad y lenguaje I. El significado como corolario cultural, Juan de Dios Luque Durán y Antonio Pamies Bertrán (eds.), pp. 235-251. Granada: Método.

- (2009): "Habla, soy todo oídos. Reflexo das accións e das actitudes do receptor na fraseoloxía somática metalingüística do español". Cadernos de fraseoloxía galega 11: 139-162.

PEÑA CERVEL, Sandra (2001): "A Cognitive Approach to the Role of Body Parts in the Conceptualization of Emotion Metaphors". EPOS XVII: 245-260.

POCH OLIVÉ, Dolors (2010): "Los poemas no existen más que en la voz", in $\mathrm{Al}$ otro lado del espejo. Comentario lingüístico de textos literarios. Estudios en homenaje a José Manuel Blecua Perdices, Gloria Clavería Nadal y Dolors Poch Olivé (coords.), pp. 187-216. Barcelona: Ariel.

PRAT SABATER, Marta (2006): "Reflejo espacial del cambio léxico: los atlas lingüísticos y el DCECH", in Actes del VII Congrés de Lingüística General (Barcelona, 18-21 abril 2006), pp. 1-15. Barcelona: Universitat de Barcelona. [Edición en CD-ROM].

[REW] MEYER-LÜBKE, Wilhelm (1968[1911]): Romanisches Etymologisches Wörterbuch, 4. ${ }^{\text {a }}$ edición. Heidelberg: Winter.

Rosch, Eleanor (1978): "Principles of Categorization", in Cognition and Categorization, Eleanor Rosch y Barbara B. Lloyd (eds.), pp. 27-48. Hillsdale (N.J.): Lawrence Erlbaum Associates.

SAnTos Domínguez, Luis A. y Rosa M. a Espinosa ElorZA (1996): Manual de semántica histórica. Madrid: Síntesis. 
SARAMAGO, João (2006): “O Atlas Lingüístico-Etnográfico de Portugal e da Galiza (ALEPG)". Estudis romànics XXVIII: 281-298.

SweETSER, Eve E. (1990): From Etymology to Pragmatics. Metaphorical and Cultural Aspects of Semantic Structure. Cambridge: Cambridge University Press.

TAGLiAvinI, Carlo (1949): "Di alcuni denominazioni della <pupila> (studio di onomasiologia, con speciale riguardo alle lingue camito-semitiche e negro africane)". Annali dell'Istituto Universitario Orientale di Napoli III: 341-378.

[TILG] SANTAMARINA FERNÁNDEZ, Antón (ed.) (2003): Tesouro informatizado da lingua galega. Santiago de Compostela: Instituto da Lingua Galega [en línea], <http://ilg.usc.es/tmilg> [Consulta: 31/10/2010].

UllmanN, Stephen (1980[1962]): Semántica. Introducción a la ciencia del significado. Madrid: Aguilar. [Traducción de Semantics: an Introduction to the Science of Meaning. Oxford: Basil Blackwell].

VAl Álvaro, José F. (1999): “La composición”, in Gramática descriptiva de la lengua española, Ignacio Bosque Muñoz y Violeta Demonte Barreto (eds.), vol. III, pp. 4757-4841. Madrid: Espasa-Calpe.

VENY ClAR, Joan (1991): “Cap a una tipologia de l'etimologia popular", in Mots d'ahir i mots d'avui, pp. 69-95. Barcelona: Empúries.

- (2000): "De la nineta a l'ànima de l'ull", in Jornades de la secció filològica de l'Institut d'Estudis Catalans a Elx i a la Universitat d'Alacant (16 i 17 d'octubre de 1998), pp. 83-92. Barcelona-Elx: Institut d'Estudis CatalansAjuntament d'Elx,

ZAUNER, Adolf (1903): "Die romanischen Namen der Körperteile". Romanische Forschungen XIV: 339-530. 